\title{
Prediction of the motor prognosis with diffusion tensor imaging in hemorrhagic stroke: a meta-analysis
}

\author{
Min Cheol Chang ${ }^{1}$, Sang Cyu Kwak ${ }^{2}$, Donghwi Park $3, *$ \\ ${ }^{1}$ Department of Rehabilitation Medicine, College of Medicine, Yeungnam University, 42415 Daegu, Republic of Korea \\ ${ }^{2}$ Department of Medical Statistics, College of Medicine, Catholic University of Daegu, 42472 Daegu, Republic of Korea \\ ${ }^{3}$ Department of Physical Medicine and Rehabilitation, Ulsan University Hospital, University of Ulsan College of Medicine, 44033 Ulsan, Republic of Korea \\ *Correspondence: bdome@uuh.ulsan.kr (Donghwi Park)
}

DOI:10.31083/j.jin2004102

This is an open access article under the CC BY 4.0 license (https://creativecommons.org/licenses/by/4.0/).

Submitted: 22 June 2021 Revised: 28 September 2021 Accepted: 27 October 2021 Published: 30 December 2021

This paper investigates whether diffusion tensor imaging performed within 2 weeks of intracerebral hemorrhage onset could predict the motor outcome by categorizing previous diffusion tensor imaging studies based on the time-point of performing diffusion tensor imaging ( $<2$ weeks and $\geq 2$ weeks after intracerebral hemorrhage onset). A comprehensive database search on PubMed, Embase, Cochrane Library, and SCOPUS was conducted. The pooled estimate was acquired using correlation analysis between the diffusion tensor imaging parameters of fractional anisotropy and motor recovery based on the period of stroke onset. In the results, out of 511 retrieved articles, eight were finally included in the meta-analysis. In patients who underwent diffusion tensor imaging within 2 weeks of intracerebral hemorrhage onset, a random-effects model revealed that the ratio of fractional anisotropy is a significant predictor of motor recovery of the hemi-side extremity after intracerebral hemorrhage ( $p=$ 0.0015 ). In patients who underwent diffusion tensor imaging after 2 weeks of intracerebral hemorrhage onset, a fixed-effects model revealed that the ratio of fractional anisotropy was also a significant predictor of motor recovery of the hemi-side extremity after intracerebral hemorrhage $(p<0.0001)$. Our meta-analysis revealed that ratio of fractional anisotropy $(\mathrm{rFa}$ ) calculated from diffusion tensor imaging (DTI) performed $\geq 2$ weeks of intracerebral hemorrhage onset had a positive correlation with the motor outcomes after intracerebral hemorrhage (ICH). Also, although diffusion tensor imaging was performed $<2$ weeks after intracerebral hemorrhage onset, the ratio of fractional anisotropy calculated from diffusion tensor imaging helped predict the motor outcome. Further analyses, including a more significant number of studies focused on this topic, are warranted.

\section{Keywords}

Diffusion tensor imaging; Intracerebral hemorrhage; Motor recovery; Stroke onset; Meta-analysis

\section{Introduction}

Intracerebral hemorrhage (ICH) is a major cause of disability in adult. Motor deficits are one of the most disabling complication in patients with ICH $[1,2]$. Over half of the patients with ICH experience motor weakness [3]. It hinders patients' independent performance of their activities of daily living and deteriorates their quality of life [1]. The corticospinal tract (CST) is one of the most important neural tracts involved in motor functions $[4,5]$. The state of CST after $\mathrm{ICH}$ is the decisive factor determining the motor prognosis $[4,5]$. Therefore, evaluating the state of CST is vital to identify the motor outcome of patients with ICH.

Diffusion tensor imaging (DTI) allows for evaluating the integrity of the neural tracts or other tissues owing to its ability to visualize the characteristics of water diffusion [6-8]. In intact neural tracts, water molecules move freely in the direction of the neural tracts. However, when the neural tracts are interrupted, the movement of water molecules is limited. Using DTI parameters, this movement, which is correlated with the integrity of the neural tracts, can be measured. Several studies showed that DTI could predict the motor outcome of patients with ICH [9-13]. In previous studies, however, the appropriate time point for obtaining DTI data has rarely been studied. Intracerebral perilesional edema is proposed to hinder an accurate analysis of DTI data because it can affect the movement of water molecules within the neural tracts [14]. Intracerebral edema after ICH is known to persist for approximately 2 weeks after the onset [15]. Therefore, in this metaanalysis study, we sought to find out the difference in motor outcome predictability of DTI based on 2 weeks of ICH occurrence.

This meta-analysis categorizes previous DTI studies into those performed $<2$ weeks and $\geq 2$ weeks after the ICH onset, thus elucidating whether DTI performed within 2 weeks after the onset could predict the motor outcome.

\section{Methods}

This meta-analysis study was conducted according to the Preferred Reporting Items for Systematic Reviews and MetaAnalysis guidelines. We searched the relevant research on SCOPUS, PubMed, Embase, and Cochrane Library for studies published until January 31, 2020. The following keywords were used in the database search: "diffusion tensor imaging", "motor recovery" and "Intracerebral hemorrhage" [11]. 


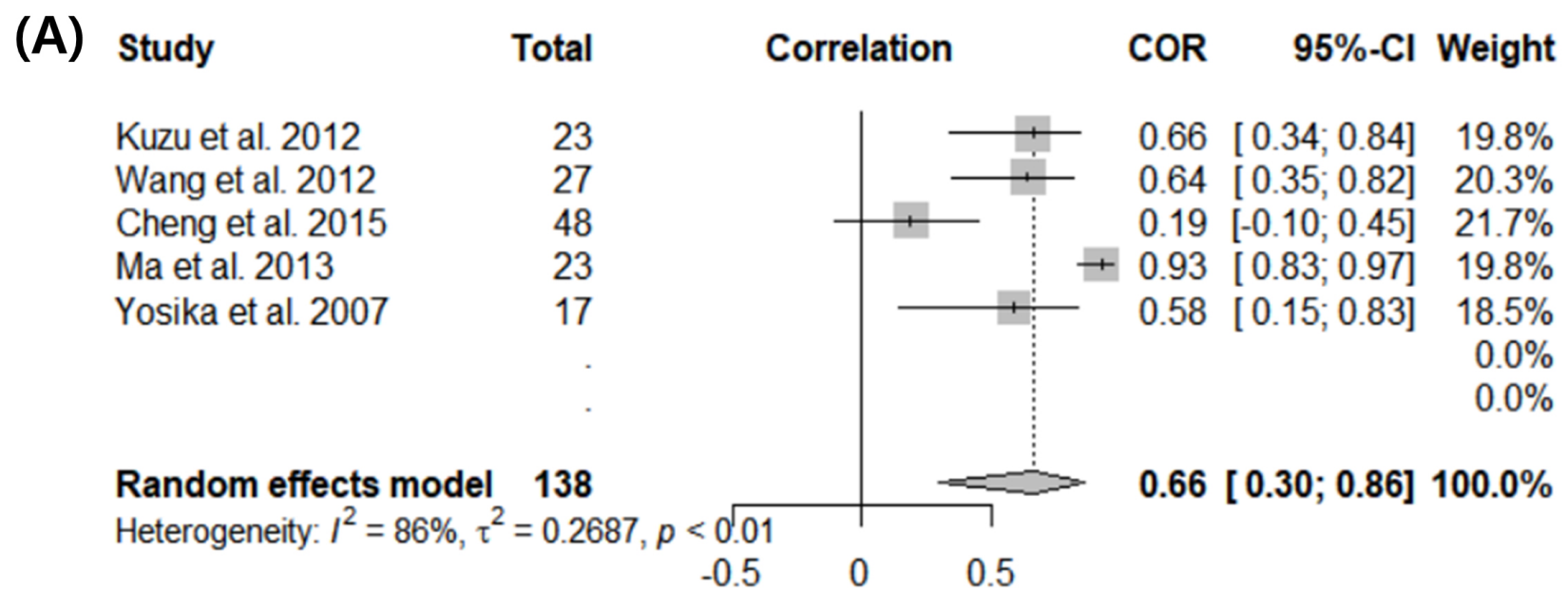

(B) Study

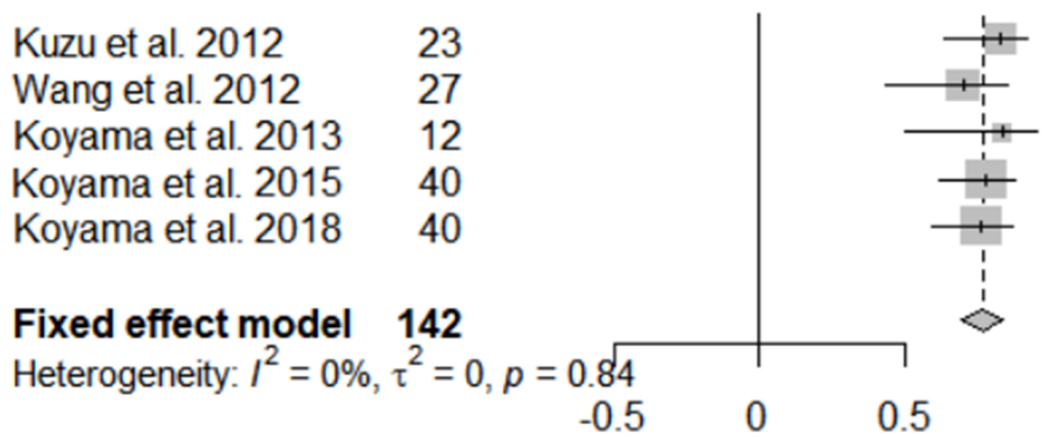

COR $\quad 95 \%-\mathrm{Cl}$ Weight

$0.83[0.64 ; 0.93] 15.7 \%$

$0.70[0.44 ; 0.85] \quad 18.9 \%$

$0.83[0.50 ; 0.95] \quad 7.1 \%$

$0.78 \quad[0.62 ; 0.88] \quad 29.1 \%$

$0.76[0.59 ; 0.87] \quad 29.1 \%$

$0.77[0.70 ; 0.84] 100.0 \%$

Fig. 1. Forest plots showing the results of (A) the correlation between ratio of fractional anisotropy (rFA) and hemi-side motor recovery in patients $<2$ weeks of intracerebral hemorrhage (ICH) onset and (B) the correlation between rFA and hemi-side motor recovery in patients $\geq 2$ weeks after ICH onset.

Filters were applied to extract studies with human subjects. We only included research published in English. A study was selected to include patients with ICH with unilateral extremity weakness who underwent DTI and follow-up assessment of clinical recovery.

Inclusion criteria included: (i) Published original study; (ii) Observational study that evaluated fractional anisotropy (FA), assessed at baseline, and its relationship with the recovery of weakness, assessed at a future time point $(\geq 3$ months after ICH onset); (iii) Study measuring FA in the areas with a course of CST; (iv) Study including patients with unilateral weakness following ICH; (v) Information about when DTI was taken was presented; (vi) Study assessing outcomes including the motor function of hemi-side extremities/functional impairment or improvement.

Exclusion criteria included: (i) Studies reporting no extractable data for independent parameters; (ii) Studies investigating responses to treatment with DTI; (iii) Case reports, case series, and review articles; (iv) Studies including patients with chronic phase ICH (inclusion of patients with ICH persisting for over 1 month).

Using a standardized data collection form, data were independently extracted by two researchers (DP and MCC). Discrepancies were resolved after rechecking the source papers and further discussions among all authors. In the three casecontrol and five cohort studies, the data of 280 patients with ICH were extracted. To assess the effectiveness of DTI for predicting motor function based on the period of stroke onset, we divided the extracted data based on the timing of DTI (DTI performed $<2$ weeks of the stroke onset and $\geq 2$ weeks of the stroke onset).

We investigated the following data from eligible research: year of publication, surname of the first author, number of patients, sex ratio, mean age, hemisphere affected, timing of DTI, lesion location, hemi-side motor power or function scale used, DTI parameters, location of FA, and duration of follow-up. We also extracted imaging parameters, including the acquisition matrix, repetition time, echo time, direction 
Table 1. Characteristics of the studies included in this meta-analysis.

Study Country No. of patients Lesion location Hemisphere R/L Age (mean \pm SD) Sex (M/F) Motor scale DTI parameter Location of FA Timing of DTI after Follow-up (days) Correlation

\begin{tabular}{|c|c|c|c|c|c|c|c|c|c|c|c|c|}
\hline Kuzu et al. 2012 [18] & Japan & 23 & $\mathrm{Pt}, \mathrm{Th}$ & & $65.0 \pm 8.1$ & $12 / 11$ & NIHSS & FA & cerebral peduncle & 3,14 & 90 & $0.661,0.830$ \\
\hline Wang et al. 2012 [19] & China & 27 & $\mathrm{Pt}, \mathrm{Th}, \mathrm{IVH}$ & $14 / 13$ & $60.2 \pm 10.5$ & $14 / 13$ & mNIHSS & FA & cerebral peduncle & 3,14 & 180 & $0.641,0.700$ \\
\hline Cheng et al. 2015 [20] & Taiwan & 48 & Pt, Th, or both & $27 / 21$ & $62.0 \pm 14.0$ & $31 / 17$ & Motricity index & FA & cerebral peduncle & $7 \pm 5$ & 90 & 0.189 \\
\hline Ma et al. 2013 [21] & China & 23 & BG and/or IVH & $11 / 12$ & $54.3 \pm 9.1$ & $15 / 8$ & NIHSS & FA & cerebral peduncle & 0 & 90 & 0.926 \\
\hline Yosika et al. 2007 [22] & Japan & 17 & $\mathrm{Pt}, \mathrm{Th}$ & & $61.8 \pm 7.6$ & $12 / 5$ & MMT & FA & cerebral peduncle & $3.17 \pm 1.33$ & 90 & 0.585 \\
\hline Koyama et al. 2013 [23] & Japan & 12 & $\mathrm{Pt}, \mathrm{Th}$ & $6 / 6$ & $62.9 \pm 14.6$ & $6 / 6$ & Brunnstrom stage & FA & cerebral peduncle & $14-18$ & -(long-term) & 0.834 \\
\hline Koyama et al. 2015 [24] & Japan & 40 & $\mathrm{Pt}, \mathrm{Th}$ & $18 / 22$ & $61.8 \pm 12.9$ & $29 / 11$ & Brunnstrom stage & FA & cerebral peduncle & $14-21$ & -(long-term) & 0.779 \\
\hline Koyama et al. 2018 [25] & Japan & 40 & $\mathrm{Pt}, \mathrm{Th}$ & & $61.0 \pm 16.7$ & $29 / 11$ & Brunnstrom stage & FA & cerebral peduncle & $14-21$ & -(long-term) & 0.763 \\
\hline
\end{tabular}

Abbreviations: Pt, putamen; T, thalamus; IVH, intraventricular hemorrhage; BG, basal ganglia; MMT, manual muscle test; FA, fractional anisotropy; DTI, diffusion tensor imaging; M/F, male/female.

Table 2. Details of imaging parameters of the studies included in this meta-analysis.

\begin{tabular}{|c|c|c|c|c|c|c|c|c|c|c|c|c|}
\hline Study & MRI system & $\begin{array}{l}\text { Acquisition } \\
\text { matrix }\end{array}$ & $\begin{array}{l}\text { Echo time } \\
(\mathrm{ms})\end{array}$ & $\begin{array}{l}\text { Repetition } \\
\text { time (ms) }\end{array}$ & $\mathrm{FOV}(\mathrm{mm})$ & $\begin{array}{l}\text { b value } \\
\left(\mathrm{s} / \mathrm{mm}^{2}\right)\end{array}$ & $\begin{array}{c}\text { Slice } \\
\text { thickness }\end{array}$ & No. of slice & Imaging software & $\begin{array}{c}\text { DTI } \\
\text { direction }\end{array}$ & ROI area & $\begin{array}{c}\text { Hematoma } \\
\text { volume }(\mathrm{mL})\end{array}$ \\
\hline $\begin{array}{l}\text { Kuzu et al. } \\
2012[18]\end{array}$ & $\begin{array}{l}\text { 3.0-tesla scanner (Signa VH/i; General } \\
\text { Electric Medical Systems, Milwaukee, }\end{array}$ & $512 \times 384$ & 25 & 4000 & 240 & & 6.5 & & $\begin{array}{c}\text { Functoll TM imaging analysis } \\
\text { software (General Electric Medical }\end{array}$ & & $\mathrm{CP}$ & $16.7 \pm 11.1$ \\
\hline
\end{tabular}

Electric Medical Systems, Milwaukee, WI, USA) Systems, Buc, France)

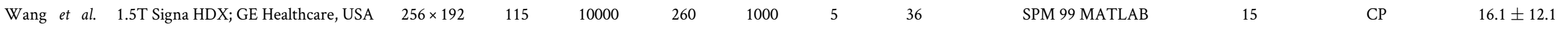
2012 [19]

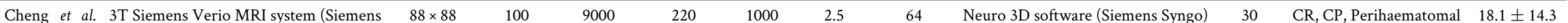

2015 [20] Medical System, Erlangen, Germany)

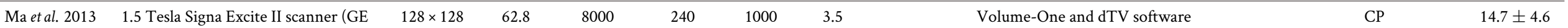

[21] Healthcare, Milwaukee, WI, USA)

Yosika et al $1.5 T$ Signa

2007 [22]

\begin{tabular}{|c|c|c|c|c|c|c|c|c|c|c|c|}
\hline $\begin{array}{l}\text { Koyama et al. } \\
2013[23]\end{array}$ & $\begin{array}{l}\text { 3T MR Scanner (Trio; Siemens AG, } \\
\text { Erlangen, Germany) }\end{array}$ & $128 \times 128$ & 83 & 7000 & 230.4 & 1000 & 3 & 64 & $\begin{array}{l}\text { FSL (analysis group, FMRIB, } \\
\text { Oxford, UK) }\end{array}$ & NA & $\mathrm{CP}$ \\
\hline $\begin{array}{l}\text { Koyama et al. } \\
2015[24]\end{array}$ & $\begin{array}{l}\text { 3T MR Scanner (Trio; Siemens AG, } \\
\text { Erlangen, Germany) }\end{array}$ & $128 \times 128$ & 83 & 7000 & 230.4 & 1000 & 3 & 64 & $\begin{array}{l}\text { FSL (analysis group, FMRIB, } \\
\text { Oxford, UK) }\end{array}$ & NA & $\mathrm{CP}$ \\
\hline Koyama et al. & 3T MR Scanner (Trio; Siemens AG, & $128 \times 128$ & 83 & 7000 & 230.4 & 1000 & 3 & 64 & FSL (analysis group, FMRIB, & NA & $\mathrm{CP}$ \\
\hline
\end{tabular}

Oxford, UK)

Abbreviations: CR, corona radiata; CP, cerebral peduncle; IC, internal capsule; BG, basal ganglia; FOV, field of view; FA, fractional anisotropy; ROI, region of interest; T, tesla; MRI, magnetic resonance imaging; NA, not 
of DTI, field of view, b-value, magnetic resonance imaging system used, slice thickness, imaging software, number of slices, hematoma volume, ratio of FA ( $\mathrm{rFA}$ ), and region of interest (ROI) area.

The quality of the included research was evaluated using the Methodological Index for Non-Randomized Studies (MINORS) [16]. Each research was measured according to 8 criteria (maximum score 16): a clearly stated aim, endpoints appropriate to the aim of the study, inclusion of consecutive patients, unbiased assessment of the study endpoint, prospective collection of data, follow-up period appropriate to the aim of the study, loss to follow-up of less than 5\%, and prospective calculation of the study. The quality of each study was categorized as reported and adequate (2), inadequate (1), and not reported (0).

The "R" statistical software version 2.2.1 (R Project for Statistical Computing, Vienna, Austria) was used to analyze the pooled data [17]. A heterogeneity test was conducted using $\mathrm{I}^{2}$ statistics, which measures the extent of inconsistency among results in each analysis. $p \geq 0.05$ indicated homogeneous pooled data, and a fixed-effects model was applied. Conversely, $p<0.05$ indicated substantial heterogeneity, and a random-effects model was used in the data analysis. We analyzed the summary of the correlation coefficient to assess the usefulness of DTI for predicting motor function. Based on the different heterogeneity levels of the rate ratio outcomes, the random-effects and fixed-effects models were selected. Further, in the analysis, a 95\% confidence interval (CI) was used. $p<0.05$ was considered statistically significant.

\section{Results}

Five hundred eleven published studies were found in the search after applying the inclusion and exclusion criteria (Fig. 1). Out of the 511 retrieved articles, eight were finally included in the meta-analysis [18-25]. Tables 1 [18-25] and 2 [18-25] demonstrate the general characteristics of the included studies, detailed methodologies, and DTI imaging parameters.

The eight studies included 280 patients with ICH with an individual sample size of 12 to 48 patients. Measurement parameters of DTI included FA and apparent diffusion coefficient. Discrepancies emerged in the regions of interest of the DTI evaluation in the included research. The included studies also used various hemi-side extremity motor outcome measures, such as the motor function scale, modified $\mathrm{Na}$ tional Institutes of Health Stroke Scale, manual muscle test, Brunstrom scale, and motricity index. Based on the timing of DTI, 138 patients with ICH derived from five studies were classified into the first group (DTI had been performed within 2 weeks of the ICH onset), and 142 patients derived from five studies were classified into the second group (DTI had been performed after 2 weeks of the ICH onset). Two studies (Kuzu et al. [18] and Wang et al. [19]) were included in both groups.
In all included research, "follow-up period appropriate to the aim of the study", "unbiased assessment of the study endpoint", "endpoints appropriate to the aim of the study", "inclusion of consecutive patients" and "a clearly stated aim" were adequately reported. Regarding "prospective collection of data”, however, Koyama et al.'s 3 studies [23-25] did not report any related content. Additionally, in 6 studies other than Cheng et al. [20] and Yosika et al. [22], information on the follow-up loss was not presented, or follow-up loss exceeded 5\% (Table 3 [18-25]). Furthermore, included research did not estimate the appropriate sample size prior to the initiation of each research.

In patients who underwent DTI within 2 weeks of the ICH onset, a random-effects model was used (heterogeneity: $\mathrm{I}^{2}=$ $86 \%, p<0.01$ ), and rFA was a significant predictor of motor recovery of hemi-side extremity after ICH (correlation coefficient $=0.66$; $95 \%$ CI: 0.30 to $0.86, p=0.0015$, effect size $=1.76$; 95\% CI: 1.31 to 2.20 ) (Fig. $1 \mathrm{~A}$ ). In patients who underwent DTI after 2 weeks of the ICH onset, however, a fixed-effects model was used (heterogeneity: $\mathrm{I}^{2}=0 \%, p=0.84$ ), and rFA was also a significant predictor of motor recovery of hemiside extremity after ICH (correlation coefficient $=0.77 ; 95 \%$ CI: 0.68 to $0.82, p<0.0001$, effect size $=2.41 ; 95 \%$ CI: 1.90 to 2.93) (Fig. 1B). Additionally, as a sensitivity test, the random effects model was also used in patients who underwent DTI after 2 weeks of the ICH onset.

Two of the authors (MCC and DP) individually evaluated the risk of bias with respect to predicting the motor prognosis with DTI based on a few distinct methods. The risk of publication bias was decided using an Egger's test and funnel plot. A funnel plot was made to evaluate the risk of publication bias (Fig. 2). The FA and motor recovery funnel plot showed some asymmetry in patients who underwent DTI within 2 weeks of the ICH onset. However, Egger's test showed no statistical significance $(p=0.2172)$, indicating that the observation of asymmetry was not supported and that there may not be a risk of publication bias. The funnel plot between FA and motor recovery showed symmetry in patients who underwent DTI 2 weeks after the ICH onset. Additionally, Egger's test was not significant $(p=0.4286)$, indicating that there may not be a risk of publication bias.

\section{Discussion}

In the current meta-analysis, we evaluated whether DTI, performed $<2$ weeks of the onset of $\mathrm{ICH}$, can predict motor outcomes after the subacute phase of stroke. We found that rFA of affected and unaffected CSTs from DTI performed $<2$ weeks of onset had a positive correlation with motor outcomes in the chronic phase of ICH. Similarly, rFA calculated from DTI performed $\geq 2$ weeks of ICH also showed a positive correlation with the motor outcomes after ICH.

The rFA value indicates the degree of directionality of water diffusion and ranges from 0 (completely isotropic diffusion) to 1 (completely anisotropic diffusion) $[26,27]$. Water diffuses easily along the neural tracts, and its diffusion is hin- 
Table 3. The result of quality assessment for selected studies.

\begin{tabular}{|c|c|c|c|c|c|c|c|c|}
\hline & $\begin{array}{l}\text { Kuzu et al. } \\
2012 \text { [18] }\end{array}$ & $\begin{array}{l}\text { Wang et al. } \\
2012 \text { [19] }\end{array}$ & $\begin{array}{l}\text { Cheng et al. } \\
2015 \text { [20] }\end{array}$ & $\begin{array}{l}\text { Ma et al. } \\
2013 \text { [21] }\end{array}$ & $\begin{array}{c}\text { Yosika et al. } \\
2007 \text { [22] }\end{array}$ & $\begin{array}{c}\text { Koyama et al. } \\
2013 \text { [23] }\end{array}$ & $\begin{array}{c}\text { Koyama et al. } \\
2015 \text { [24] }\end{array}$ & $\begin{array}{c}\text { Koyama et al. } \\
2018 \text { [25] }\end{array}$ \\
\hline A clearly stated aim & 2 & 2 & 2 & 2 & 2 & 2 & 2 & 2 \\
\hline Inclusion of consecutive patients & 2 & 2 & 2 & 2 & 2 & 2 & 2 & 2 \\
\hline Prospective collection of data & 2 & 2 & 2 & 2 & 2 & 0 & 0 & 0 \\
\hline Endpoints appropriate to the aim of the study & 2 & 2 & 2 & 2 & 2 & 2 & 2 & 2 \\
\hline Unbiased assessment of the study endpoint & 2 & 2 & 2 & 2 & 2 & 2 & 2 & 2 \\
\hline Follow-up period appropriate to the aim of the study & 2 & 2 & 2 & 2 & 2 & 2 & 2 & 2 \\
\hline Loss to follow up less than $5 \%$ & 0 & 1 & 2 & 0 & 2 & 0 & 0 & 0 \\
\hline Prospective calculation of the study size & 0 & 0 & 0 & 0 & 0 & 0 & 0 & 0 \\
\hline Total & 12 & 13 & 14 & 12 & 14 & 10 & 10 & 10 \\
\hline
\end{tabular}

(A)

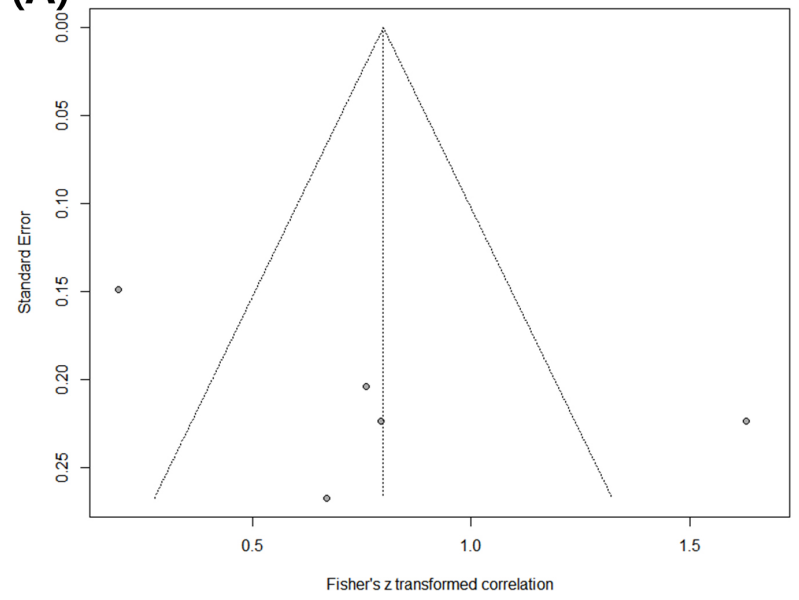

(B)

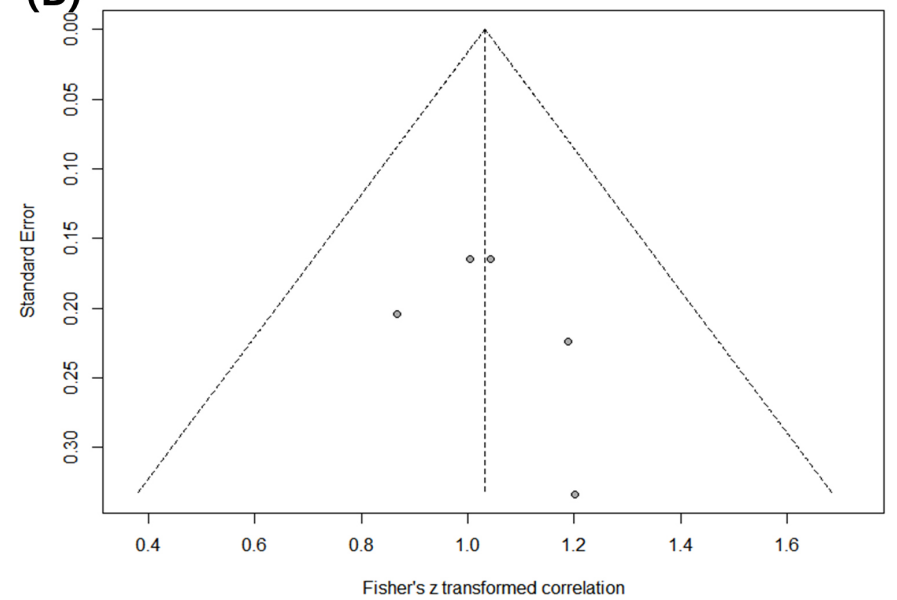

Fig. 2. Funnel plots of the meta-analysis. (A) Funnel plots for predicting a hemi-side motor recovery in patients within 2 weeks of intracerebral hemorrhage (ICH) onset. (B) Funnel plots for the prediction of hemi-side motor recovery in patients 2 weeks after ICH onset.

dered within the damaged neural tracts [26, 27]. Therefore, while an increased FA value reflects the intactness (or preservation) of neural tracts, a decreased FA value indicates deterioration $[26,27]$. In the field of DTI, the FA value is most commonly used for investigating the degree of directionality of the microstructure (e.g., axon, myelin, and microtubules) of neural tracts in patients with stroke or traumatic brain injury $[2,4,5,28]$.

However, intra-myelin edema at an early stage of ICH can cause the intra-neural water to move to be more parallel to the neural tract. Because vasogenic edema surrounding the intracerebral hematoma is known to persist for up to 2 weeks after onset, some researchers thought that DTI performed at an early stage of brain injury is inaccurate in predicting the motor prognosis $[14,15]$. However, in our study, although the FA value of CST in the affected hemisphere was calculated from DTI performed within 2 weeks after onset, it positively correlated with motor outcomes. Additionally, the correlation coefficient was 0.66 , which indicates that the $\mathrm{rFA}$ value and motor outcomes after the subacute phase of ICH have a moderate correlation [29]. We expect that the effect of edema on the FA value might not be significant. However, considering that the correlation coefficient between the rFA value measured on DTI performed $\geq 2$ weeks after onset and motor outcome was 0.76 (strong correlation) [29].

Our meta-analysis revealed that although DTI was performed $<2$ weeks after ICH onset, rFA calculated from DTI could help predict motor outcomes. However, there may be greater risk of publication bias in the studies that examined $\mathrm{rFA}$ and motor outcomes in the two weeks following $\mathrm{ICH}$, which means results need to be interpreted cautiously. We should consider the possibility that researchers did not report their findings when rFA measured from DTI taken within 2 weeks after stroke was not correlated with motor recovery. Our study is the first meta-analysis performed by dividing previous studies based on when DTI was performed. Our study could be helpful for clinicians to interpret the results of DTI based on the time at which DTI was performed. Our study is limited in that the number of included studies in the meta-analysis was relatively small. Additionally, the assessment of motor outcomes by different scales in the included studies is a serious limitation, due to the degree of 
heterogeneity across studies regarding outcome assessments. However, the relatively large number of patients compared to the small number of included studies is considered to be the strength of our study, although there may be some participant overlaps because two of the included studies examine DTI at 3 days after ICH and 14 days after ICH $[18,19]$.

Additionally, the assessment of motor outcomes by different scales in the included studies is a severe limitation due to heterogeneity across studies regarding outcome assessments. However, as a meta-analysis study investigating the importance of DTI imaging timing for predicting a motor recovery in ICH patients, we think these results are valuable. In the future, further studies on this topic with large numbers of patients using the same protocol are warranted. Moreover, it would be interesting to study the accuracies of the DTI analysis at multiple time points within 2 weeks after ICH onset (e.g., $<2-3$ days and $<1$ week).

\section{Author contributions}

MCC-study concept and design, acquisition of data, revision of manuscript and critical revision of manuscript for intellectual content. SGK-analysis of data. DP-study concept and design, analysis of data, and critical revision of manuscript for intellectual content.

\section{Ethics approval and consent to participate Not applicable.}

\section{Acknowledgment \\ Not applicable.}

\section{Funding}

The present study was supported by a National Research Foundation of Korea grant funded by the Korean government (grant no. NRF-2019M3E5D1A02069399).

\section{Conflict of interest}

The authors declare no conflict of interest.

\section{References}

[1] Saulle MF, Schambra HM. Recovery and Rehabilitation after Intracerebral Hemorrhage. Seminars in Neurology. 2016; 36: 306312.

[2] Jang SH, Choi BY, Chang CH, Kim SH, Chang MC. Prediction of motor outcome based on diffusion tensor tractography findings in thalamic hemorrhage. International Journal of Neuroscience. 2013; 123: 233-239.

[3] Jang SH, Ahn SH, Sakong J, Byun WM, Choi BY, Chang CH, et al. Comparison of TMS and DTT for predicting motor outcome in intracerebral hemorrhage. Journal of the Neurological Sciences. 2010; 290: 107-111.

[4] Kwak SY, Kwak SG, Yoon TS, Kong EJ, Chang MC. Deterioration of Brain Neural Tracts in Elderly Women with Sarcopenia. The American Journal of Geriatric Psychiatry. 2019; 27: 774-782.

[5] Moon JS, Chung SM, Jang SH, Won KC, Chang MC. Effects of Diabetes on Motor Recovery after Cerebral Infarct: a Diffusion Tensor Imaging Study. The Journal of Clinical Endocrinology \& Metabolism. 2019; 104: 3851-3858.
[6] Razek AAKA, El-Serougy L, Abdelsalam M, Gaballa G, Talaat M. Differentiation of residual/recurrent gliomas from postradiation necrosis with arterial spin labeling and diffusion tensor magnetic resonance imaging-derived metrics. Neuroradiology. 2018; 60: $169-177$.

[7] El-Serougy L, Abdel Razek AAK, Ezzat A, Eldawoody H, El-Morsy A. Assessment of diffusion tensor imaging metrics in differentiating low-grade from high-grade gliomas. The Neuroradiology Journal. 2016; 29: 400-407.

[8] Abdel Razek AAK. Routine and Advanced Diffusion Imaging Modules of the Salivary Glands. Neuroimaging Clinics of North America. 2018; 28: 245-254.

[9] Puig J, Blasco G, Schlaug G, Stinear CM, Daunis-I-Estadella $\mathrm{P}$, Biarnes $\mathrm{C}$, et al. Diffusion tensor imaging as a prognostic biomarker for motor recovery and rehabilitation after stroke. Neuroradiology. 2017; 59: 343-351.

[10] Groisser BN, Copen WA, Singhal AB, Hirai KK, Schaechter JD. Corticospinal tract diffusion abnormalities early after stroke predict motor outcome. Neurorehabilitation and Neural Repair. 2014; 28: 751-760.

[11] Kumar P, Yadav AK, Misra S, Kumar A, Chakravarty K, Prasad K. Prediction of upper extremity motor recovery after subacute intracerebral hemorrhage through diffusion tensor imaging: a systematic review and meta-analysis. Neuroradiology. 2016; 58: 1043-1050.

[12] Novakovic N, Wilseck ZM, Chenevert TL, Xi G, Keep RF, Pandey AS, et al. Assessing early erythrolysis and the relationship to perihematomal iron overload and white matter survival in human intracerebral hemorrhage. CNS Neuroscience \& Therapeutics. 2021; 27: 1118-1126.

[13] Novakovic N, Linzey JR, Chenevert TL, Gemmete JJ, Troost JP, Xi $\mathrm{G}$, et al. White Matter Survival within and around the Hematoma: Quantification by MRI in Patients with Intracerebral Hemorrhage. Biomolecules. 2021; 11.

[14] Edlow BL, Copen WA, Izzy S, Bakhadirov K, van der Kouwe A, Glenn MB, et al. Diffusion tensor imaging in acute-to-subacute traumatic brain injury: a longitudinal analysis. BMC Neurology. 2016; 16: 2.

[15] Zheng H, Chen C, Zhang J, Hu Z. Mechanism and Therapy of Brain Edema after Intracerebral Hemorrhage. Cerebrovascular Diseases. 2016; 42: 155-169.

[16] Slim K, Nini E, Forestier D, Kwiatkowski F, Panis Y, Chipponi J. Methodological index for non-randomized studies (minors): development and validation of a new instrument. ANZ Journal of Surgery. 2003; 73: 712-716.

[17] Carter KW, Francis RW, Carter KW, Francis RW, Bresnahan M, Gissler M, et al. ViPAR: a software platform for the Virtual Pooling and Analysis of Research Data. International Journal of Epidemiology. 2016; 45: 408-416.

[18] Kuzu Y, Inoue T, Kanbara Y, Nishimoto H, Fujiwara S, Ogasawara $\mathrm{K}$, et al. Prediction of motor function outcome after intracerebral hemorrhage using fractional anisotropy calculated from diffusion tensor imaging. Cerebrovascular Diseases. 2012; 33: 566-573.

[19] Wang D, Li J, Liu J, Hu H. Diffusion tensor imaging predicts longterm motor functional outcome in patients with acute supratentorial intracranial hemorrhage. Cerebrovascular Diseases. 2012; 34: 199-205.

[20] Cheng C, Hsu C, Huang Y, Tsai Y, Hsu H, Yang W, et al. Motor outcome of deep intracerebral haemorrhage in diffusion tensor imaging: comparison of data from different locations along the corticospinal tract. Neurological Research. 2015; 37: 774-781.

[21] Ma C, Liu A, Li Z, Zhou X, Zhou S. Longitudinal study of diffusion tensor imaging properties of affected cortical spinal tracts in acute and chronic hemorrhagic stroke. Journal of Clinical Neuroscience. 2014; 21: 1388-1392.

[22] Yoshioka H, Horikoshi T, Aoki S, Hori M, Ishigame K, Uchida $\mathrm{M}$, et al. Diffusion tensor tractography predicts motor functional outcome in patients with spontaneous intracerebral hemorrhage. Neurosurgery. 2008; 62: 97-103. 
[23] Koyama T, Marumoto K, Miyake H, Ohmura T, Domen K. Relationship between diffusion-tensor fractional anisotropy and longterm outcome in patients with hemiparesis after intracerebral hemorrhage. NeuroRehabilitation. 2013; 32: 87-94.

[24] Koyama T, Uchiyama Y, Domen K. Associations of DiffusionTensor Fractional Anisotropy and FIM Outcome Assessments after Intracerebral Hemorrhage. Journal of Stroke and Cerebrovascular Diseases. 2018; 27: 2869-2876.

[25] Koyama T, Marumoto K, Uchiyama Y, Miyake H, Domen K. Outcome assessment of hemiparesis due to intracerebral hemorrhage using diffusion tensor fractional anisotropy. Journal of Stroke and Cerebrovascular Diseases. 2015; 24: 881-889.
[26] Assaf Y, Pasternak O. Diffusion Tensor Imaging (DTI)-based White Matter Mapping in Brain Research: a Review. Journal of Molecular Neuroscience. 2008; 34: 51-61.

[27] Neil JJ. Diffusion imaging concepts for clinicians. Journal of Magnetic Resonance Imaging. 2008; 27: 1-7.

[28] Zhu J, Ling J, Ding N. Association between Diffusion Tensor Imaging Findings and Cognitive Outcomes Following Mild Traumatic Brain Injury: a PRISMA-Compliant Meta-Analysis. ACS Chemical Neuroscience. 2019; 10: 4864-4869.

[29] Rensink RA. The nature of correlation perception in scatterplots. Psychonomic Bulletin \& Review. 2017; 24: 776-797. 